\title{
Relación entre autoconcepto y nivel de depresión en personas con retinosis pigmentaria
}

\author{
Helena Chacón-López* y M. Dolores López-Justicia
}

Universidad de Granada (España).

\begin{abstract}
Resumen: Investigaciones previas han resaltado dificultades en autoconcepto y presencia de depresión en personas con una patología degenerativa visual (retinosis pigmentaria), pero no se conoce si existe relación entre estas variables, la estimación del número de personas de apoyo en caso de necesidad y la edad; siendo éste el primer objetivo del estudio, realizado con 51 personas $(36.5 \%$ hombres y $63.5 \%$ mujeres $)(M$ edad $=41.85)$ que padecen esta problemática; y determinar, en segundo lugar, si el nivel de depresión influye en el autoconcepto. Se utilizó la Escala de Autoconcepto Tennesse (Fitts, 1965), el Inventario de Depresión de Beck (Beck, Rush, Shaw, y Emery, 1979) y un documento sociodemográfico. La correlación de Pearson revela que el número de personas de apoyo correlaciona con los factores de autoconcepto físico y personal, la edad con puntuaciones más altas en depresión, asociándose ésta a menores puntuaciones justo en los dos factores mencionados. El MANCOVA efectuado confirma que las personas con depresión se percibían más negativamente en todos los factores de autoconcepto. Se concluye recomendando prevenir la depresión y favorecer la mejora del autoconcepto con el fin de potenciar la competencia personal, calidad de vida y funcionamiento visual de esta población.

Palabras clave: Autoconcepto; depresión; degeneración retiniana; retinosis pigmentaria; adultos.
\end{abstract}

\section{Introducción}

En los últimos años ha aumentado el interés en el estudio de las dificultades personales y emocionales de afectados por una enfermedad progresiva y degenerativa del sistema visual, como la retinosis pigmentaria (RP), permitiendo conocer algo mejor las limitaciones que conlleva y mitigar la escasez de investigación en el ámbito psicológico-educativo. Escasez atribuida probablemente a la baja incidencia de esta patología, aunque constituye la cuarta causa más habitual de ceguera, habiéndose estimado en España alrededor de 25.000 personas afectadas y 500.000 posibles transmisoras de la misma (Fernández, 2007; Hamel, 2006). La RP es una enfermedad retiniana para la que no existen aún soluciones médicas o tratamientos farmacológicos, suele afectar a los dos ojos y tiene importantes consecuencias en la vida adulta al producirse un grave deterioro visual, que puede llegar a la ceguera (Agurtzane y Vecino, 2009; Fernández, 2007; Rundquist, 2004). Generalmente se inicia en la infancia pero es alrededor de los 20 años cuando, frecuentemente, empieza a ser más incapacitante visualmente (Chacón-López, LópezJusticia, et al., 2013; López-Justicia, Polo, et al., 2011). Su incidencia aproximada es de 1 por cada 3000-5000 personas, siendo algo más frecuente en hombres $(65 \%)$ que en mujeres (55\%) (Fernández, 2007; Velvet, Gasper, Eisenacher, y Wittinghofer, 2008). El avance y progresión de la misma es va-

* Dirección para correspondencia [Correspondence address]:

Dra. Helena Chacón-López. Facultad de Ciencias de la Educación. Departamento de Psicología Evolutiva y de la Educación. Universidad de Granada. Campus Universitario de La Cartuja, s/n. 18071, Granada (España).E-mail: helenachacon@ugr.es
Title: Relationship between self-concept and depression level in people with Retinitis Pigmentosa.

Abstract: Previous studies have highlighted difficulties in self-concept and the presence of depression in people with a degenerative visual pathology visual (Retinitis Pigmentosa), but it is not known if there is a relationship between these variables, the estimate of the number of support people in case of need and age; this being the first objective of the study, carried out with 51 people affected $(36.5 \%$ men and $63.5 \%$ women $)(M$ age $=41.85)$; and secondly, to analyze if depression level could influence some selfconcept factors. Instruments such as the Tennessee Self-Concept Scale (Fitts, 1965), the Beck Depression Inventory (Beck, Rush, Shaw, \& Emery, 1979) and a sociodemographic questionnaire were used. A Pearson's correlation analysis revealed that the number of support people correlates with the factors of physical and personal self-concept, age is associated with higher scores on depression, associating this to lower scores in the two factors above-mentioned. The MANCOVA made confirms that people with higher level of depression were perceived more negatively in all selfconcept factors. It concludes recommending to prevent depression and to promote the improvement of the self-concept, in order to enhance the personal competence, the quality of life and visual functioning of this people. Key words: Self-concept; depression; retinal degeneration; retinitis pigmentosa; adults.

riable, aunque paulatinamente va asociando limitaciones en la realización de determinadas tareas cotidianas, provocando que un buen número de afectados se perciban más negativamente (Kiser y Dagnelie, 2008), por sus dificultades crecientes en la práctica de éstas, o en el desenvolvimiento por el espacio (Fuhr, Liu, y Kuyk, 2007; Rundquist, 2004).

Entre los temas que han despertado el interés investigador en el ámbito psicológico con esta población se halla el estudio del autoconcepto, al considerar que juega un papel importante en la personalidad y es un indicador de bienestar psicológico (Fernández, López-Justicia y Polo, 2007; LópezJusticia, Fernández-Castillo, Fernández y Polo, 2011; LópezJusticia y Nieto 2006); así como el análisis de su estado emocional (Chacón-López, López-Justicia, Fernández, Chacón, y Polo, 2013; Hahm et al., 2008; López-Justicia y ChacónLópez, 2015; López-Justicia, Polo, Fernández, ChacónLópez, Díaz-Batanero, y Chacón, 2011).

Algunos enfoques teóricos consideran que el autoconcepto se refiere al conjunto de características que cada persona utiliza para definirse como individuo y diferenciarse de los demás (Harter, 1999; Shavelson, Hubner, y Stanton, 1976). Asimismo, se defiende que el autoconcepto tiene un carácter jerárquico y multidimensional (González-Pienda et al., 2003; Shavelson et al., 1976). Uno de los modelos más extendidos es el propuesto por Shavelson et al. (1976) el cual plantea la existencia de un autoconcepto general, compuesto por el autoconcepto académico y no académico, incluyendo éste el social, emocional y físico. De acuerdo con este modelo el autoconcepto tiene entidad propia, posee estructura organizada, jerárquica (ocupando la parte superior de la jerarquía el autoconcepto general) y es multidimensional (aumen- 
tando ésta con la edad). Es un constructo que evoluciona con los años como resultado de la experiencia que la persona tiene en su medio social, aceptándose que, mientras el autoconcepto general suele mantenerse prácticamente inalterable (Coll, 2002), los más específicos pueden experimentar variaciones. En la edad adulta se defiende que una persona es madura en el aspecto psicológico y comportamental si se conoce, percibe y valora de modo realista, sin alteraciones muy marcadas en su autoconcepto (Fierro, 2000).

El autoconcepto es un indicador de satisfacción y bienestar psicológico que contribuye a la salud y equilibrio mental (Fuentes, García, Gracia, y Lila, 2011; Goñi, Esnaola, Rodríguez, y Camino, 2015), por lo que puntuaciones más altas en este constructo permiten enfrentarse en mejores condiciones a las consecuencias de la discapacidad, mientras que puntuaciones más bajas repercuten negativamente en la salud física y se relacionan con depresión e infravaloración personal (Smith, Nolen-Hoeksema, Fredrickson, y Loftus, 2003).

$\mathrm{Al}$ ser la RP una enfermedad progresiva, con efectos notables en la vida de las personas afectadas a medida que avanza la patología, se ha prestado atención al papel que desempeña la edad en el autoconcepto de población con edades comprendidas entre los 19 y 60 años, constatando que aquellas de más edad se sienten mejor en el ámbito mo$\mathrm{ral} /$ ético, aunque muestran mayores dificultades en el autoconcepto familiar (López-Justicia, Fernández-Castillo, et al., 2011). Dificultades también detectadas en el autoconcepto familiar de población joven afectada por idéntica patología (López-Justicia y Nieto 2006), lo que ha llevado a recomendar ayuda psicológica tanto a los afectados como a los familiares para mejorar el bienestar familiar, dado el efecto emocional negativo que causaba en éstos la presencia de RP en sus allegados (Chacón-López, López-Justicia, et al., 2013; López-Justicia, Polo, et al., 2011). El apoyo social percibido, o cómo interpreta cada cual los lazos que le unen a sus familiares o a otras personas con las que conviven, se ha relacionado con el estado de salud (Martos y Muñoz, 2011), así como con el autoconcepto en personas sin dificultades visuales (Torres, Pompa, Meza, Ancer, y González, 2010); habiéndose demostrado que la disminución de la percepción del apoyo familiar incrementa los síntomas depresivos $(\mathrm{Nu}-$ nes, Munhoz, y Ferrari, 2014).

También se han hallado diferencias entre hombres y mujeres en esta población (con edades comprendidas entre los 19 y 60 años), destacando que las mujeres se percibían mejor que los hombres en autoconcepto físico (López-Justicia, Fernández-Castillo, et al., 2011). Igualmente, se ha indagado en la influencia del nivel de estudios en el autoconcepto, concluyendo que las personas con menor nivel de estudios obtenían puntuaciones más bajas en el factor físico (LópezJusticia, Fernández-Castillo, et al., 2011).

Según la Organización Mundial de la Salud (O.M.S., 2006), la depresión es la cuarta causa de discapacidad en el mundo (de un total de seis), afectando en torno a 121 millones de personas. En relación con la RP, otras investigaciones han detectado la presencia de depresión entre los afectados
(Chacón-López, López-Justicia, et al., 2013; Hahm et al., 2008; López-Justicia, Polo, et al., 2011). Igualmente, se han encontrado alteraciones en esta variable vinculadas al avance de la edad y al sexo (López-Justicia y Chacón-López, 2015) en poblaciones con edades comprendidas entre 20 y 65 años (distribuidas en tres grupos de edad: 20-35, 36-50 y 51-65), concluyendo que el incremento de la edad se asocia a mayores niveles de depresión, sobre todo en los hombres, situándose en el nivel de depresión moderada el grupo de mayor edad. La depresión que presentan personas que padecen RP constituye la principal fuente de discapacidad funcional en tareas de la vida diaria (Hahm et al., 2008; Szlyk et al., 2001), incide negativamente en el uso de su visión al disminuir el aprovechamiento visual (Chacón-López, Pelayo, et al., 2013) y reduce su calidad de vida, independientemente del estado fisiológico y gravedad de la enfermedad (Hahm et al., 2008). Asimismo, la depresión está relacionada con peores resultados en la rehabilitación visual (Chacón-López, Pelayo, et al., 2013; Grant, Seiple, y Szlyk, 2011; Horowitz y Reinhardt, 2006), de ahí la importancia de detectarla y tratarla, para evitar sus efectos negativos en tareas visuales y en el bienestar personal de los afectados.

Aun habiéndose detectado las dificultades que presentan muchas de las personas con RP en el autoconcepto y en el ámbito emocional (que pueden reflejarse negativamente en su calidad de vida y en la ejecución en los programas de rehabilitación visual en los que participen), no se conocen estudios que hayan tratado de determinar la relación entre estas variables, la edad y la estimación del número de personas con las que creen contar en caso de necesitarlo; a pesar de la evidencia de sus limitaciones en algunos factores del autoconcepto durante la adolescencia, que se mantienen en edades avanzadas, asociándose, además, a depresión en un buen número de casos.

Con el propósito de arrojar luz a la situación descrita y aportar algunas sugerencias en la atención clínica, educativa y rehabilitadora, que requiere el colectivo, este estudio se ha marcado como objetivos: investigar la existencia de relación entre los factores del autoconcepto, la estimación y percepción del número de personas con las que creen contar en caso de necesidad (por su vinculación con el autoconcepto y con la depresión en personas sin dificultades visuales), el estado emocional (evaluando nivel de depresión) y la edad de personas aquejadas por RP; en segundo lugar, se busca determinar si el nivel de depresión de personas con RP influye en alguno o algunos factores de su autoconcepto.

\section{Método}

\section{Participantes}

La muestra la constituían 51 personas afectadas de RP con un rango de edad de entre 20 y 65 años ( $M$ edad $=$ 41.85; DT $=12.03)$, de los cuales 15 eran hombres $(36.5 \%)$ $(M$ edad $=39.38 ; D T=13.07)$ y 36 mujeres $(63.5 \%)(M$ edad $=42.45 ; D T=11.90)$. Todos tenían pérdida acentuada del 
campo visual (CV) en ambos ojos (estando situado en el momento del estudio entre $5^{\circ}$ y $20^{\circ}$ ) y de la agudeza visual (AV), o capacidad de discriminar detalles finos (Cubbigge, 2006); oscilando entre 1 y 0.05 en el mejor de sus ojos, todo ello evaluado con la escala de AV de Snellen y una campimetría digital. La Tabla 1 muestra las características sociodemográficas de los participantes.

Tabla 1. Características sociodemográficas de los participantes.

\begin{tabular}{lc}
\hline Características & Participantes \\
\hline Edad & \\
Total: $\left[\mathrm{n}^{\circ}\right] M(D T)(\%)$ & {$[51] 41.85(12.03)$} \\
Hombres & {$[15] 39.38(13.07)(36.5)$} \\
Mujeres & $42.45(11.90)(63.5)$ \\
Nivel de estudios, $\mathrm{n}^{\circ}(\%)$ & $3(5.87)$ \\
Sin estudios & $15(29.55)$ \\
Primarios & $16(31.37)$ \\
Medios & $17(33.33)$ \\
Superiores & \\
Estado civil, $\mathrm{n}^{\circ}(\%)$ & $14(27.45)$ \\
Solteros & $29(56.86)$ \\
Casados & $8(15.68)$ \\
Separados/Viudos & \\
Profesión, $\mathrm{n}^{\circ}$ (\%) & $10(19.60)$ \\
Desempleados & $14(27.45)$ \\
Empleo no cualificado & $21(41.17)$ \\
Empleo cualificado & $6(11.76)$ \\
Jubilados &
\end{tabular}

La media en años de afectación era de 14.35 (DT $=$ 10.33). Se establecieron los siguientes criterios para la inclusión en la muestra: haber sido diagnosticados al menos tres años antes de la evaluación, para evitar el mayor estrés que ocurre inmediatamente después del diagnóstico y evitar el efecto que éste pudiera tener en las respuestas; mantener resto visual, y no padecer ninguna otra enfermedad o discapacidad. Todos eran voluntarios que participaban en un proyecto coordinado por una de las autoras, que trataba de detectar posibles dificultades en el estado emocional tanto de los afectados, como del de los familiares con los que convivían. Ninguno de los participantes estaba recibiendo ningún tratamiento para la depresión o ansiedad.

\section{Instrumentos}

En primer lugar, se aplicó un documento/ficha sobre variables sociodemográficas (edad, años desde el diagnóstico, presencia de otros problemas de salud), elaborado ad hoc para este estudio. Aparte de estos datos, se incluyeron 2 ítems adicionales para determinar la percepción o estimación acerca del número de personas con las que los participantes creen contar para prestarles ayuda física y/o emocional en caso de necesitarlo: 1 ítem se refería al número de familiares, y el $2^{\circ}$ al número de amigos, o personas cercanas que creían disponibles para prestarles ayuda. La puntuación final objeto de análisis de estos 2 ítems correspondía a la media, resultante de sumar ambas respuestas.
Para evaluar el autoconcepto se utilizó la Escala de Autoconcepto Tennesse de Fitts (1965), adaptada y validada por Garanto (1984) para población española. Se eligió por haber sido usada en otros estudios con personas de características similares a las de éste y por obtener datos relativos a la estructura multidimensional del autoconcepto, aspecto considerado esencial en la actualidad, pues permite comprender mejor su relación con indicadores de salud mental (Marhs, Parada, y Ayotte, 2004). La escala está bien estandarizada, cubre un rango completo de ajuste psicológico, los datos de la fiabilidad, obtenida mediante test-retest son adecuados (.87 para la dimensión física, .80 para la dimensión moral-ética, .85 para la dimensión personal, .89 para la dimensión familiar y .90 para la dimensión social). La validez concurrente, calculada mediante correlación de Pearson con el 16 PF de Cattel (Cattel, Eber, y Tatsuoka, 1970), con una muestra de 102 personas mostraba correlaciones altamente significativas entre ambos instrumentos indicativas de fuerte consistencia interna. La validez de constructo, con una muestra de 200 personas, realizado mediante análisis factorial, hace referencia a la existencia de un factor denominado actitud hacia sí mismo, en el que saturan los componentes de la Escala de autoconcepto Tennessee.

La escala está compuesta por 100 ítems/preguntas (con respuesta tipo Likert de 5 puntos: desde 1, que indica completamente falso; hasta 5 que indica completamente cierto), 45 expresadas en forma positiva y 45 en forma negativa, las 10 preguntas restantes pertenecen a la escala L (Mentira) del Inventario Multifásico de Personalidad de Minnesotta (Minnesota Multiphasic Personality Inventory - MMPI), que está relacionado con la autocrítica. Los 90 ítems aparecen clasificados en dos dimensiones, una interna y otra externa. La dimensión interna consta de tres escalas: a) Identidad o autoconcepto; b) Autosatisfacción o autoestima; y c) Autocomportamiento. Mientras la dimensión externa consta de cinco escalas o factores: Personal (valoración de su personalidad); Familiar (cómo se siente en su familia); Moral-ético (valoración moral o ética); Social (relaciones sociales que establece); y Físico (valoración de su apariencia física); referidas cada una de éstas a tres aspectos o componentes de las actitudes hacia sí mismo. La máxima puntuación que se puede obtener en el factor personal oscila entre 46 y $84(M=65)$ para los hombres y entre 46 y $78(M=65)$ para las mujeres; en el factor físico los rangos se sitúan entre 41 y $82(M=67)$ para los hombres y entre 47 y $80(M=68)$ para las mujeres; en el factor moral/ético, entre 45 y $82(M=69)$ para los hombres y entre 55 y $82(M=70)$ para las mujeres; el factor familiar oscila entre 43 y $87(M=67)$ para los hombres y entre 54 y $82(M=71)$ para las mujeres; el factor social, entre 50 y 82 $(M=65)$ para los hombres y entre 50 y $85(M=67)$ para las mujeres.

El tiempo en que se puede completar la prueba está en torno a 13 y 20 minutos.

La evaluación del nivel de depresión fue realizada usando el Inventario de Depresión de Beck (Beck Depression Inventory - BDI) (Beck et al., 1979), instrumento autoaplicable, vali- 
dado para la población española (Vázquez y Sanz, 1997), para cuantificar los síntomas depresivos en poblaciones normales y clínicas (Sanz y Vázquez, 1998). El BDI posee una fiabilidad, en base a la consistencia interna (coeficiente alfa de Cronbach) de .83 y la estabilidad temporal (correlación testretest) oscila entre .60 y .72 (Beck, Steer, y Garbin, 1988). Los índices de validez convergente (con respecto a la escala de depresión de Zung, 1965) fueron altos (correlaciones entre .79 y .89) y la validez discriminante fue aceptable (correlación entre .11 y .45). La versión utilizada en este estudio fue la abreviada de 13 ítems, cuya consistencia interna para población psiquiátrica es de .83 y de .90 para población no psiquiátrica (Beck, et al., 1988); asimismo, la correlación entre la forma extensa y la abreviada era alta (.96) (Beck et al., 1979). Se eligió esta versión corta para facilitar la lectura a las personas con RP. En esta versión hay que elegir una frase entre un conjunto de cuatro alternativas, ordenadas por su gravedad. Cada ítem se valora con diferentes opciones de respuesta de 0 a 4, dando una puntuación total posible de 39 puntos. Se toman en consideración las siguientes puntuaciones: 0 - 4 Depresión ausente; 5 - 7 Depresión leve; 8 - 15 Depresión moderada; y superior a 15 Depresión grave Joffre-Velázquez, Martínez-Perales, García-Maldonado, y Sánchez-Gutiérrez, 2007).

\section{Procedimiento}

Todos los participantes eran voluntarios, miembros de dos Asociaciones de Retinosis Pigmentaria, que participaban voluntariamente en un proyecto con las autoras del estudio. Fueron informados de las características del estudio y se les entregó para su firma el consentimiento informado elaborado para la investigación, aprobado por el Comité de Ética de la Universidad de Granada.

En un laboratorio que reunía las condiciones necesarias de luminosidad, completaron individualmente las diferentes pruebas (adaptados todos los cuestionarios y hojas de respuestas a un tamaño de letra de 14 y 16 puntos, según las necesidades), en una única sesión. Primero se les facilitó el documento/ficha de variables sociodemográficas, seguido de la Escala de Autoconcepto Tennesse de Fitts (1965), finalizando con el BDI (Beck et al., 1979). Todas las pruebas fueron aplicadas por una investigadora especialmente entrenada para tal fin, miembro del proyecto coordinado por una de las autoras, asegurándose de la cumplimentación adecuada de todos los instrumentos.

Para evitar posibles sesgos en la recogida de los datos se adoptó la siguiente medida: se informó a los participantes de las características del estudio de forma general no pormenorizada, con el propósito de no ofrecer pistas que pudieran influir en las respuestas en la dirección de las hipótesis de la investigación.

\section{Análisis estadísticos}

$\mathrm{El}$ análisis de los datos fue realizado usando el paquete estadístico SPSS versión 20.0. para Windows. En la primera fase de la investigación, realizada con todo el grupo en su conjunto, se llevó a cabo un análisis de correlación de Pearson con el fin de conocer la posible relación entre los distintos factores de autoconcepto (Personal, Familiar, Moralético, Social y Físico), puntuaciones en depresión, edad y estimación del número de personas de apoyo (con las que cuentan en caso de necesidad). Puesto que los años transcurridos desde el diagnóstico podrían tener alguna relación se incluyó en el análisis.

En segundo lugar se efectuó un análisis de regresión lineal para determinar qué factor o factores de autoconcepto podían ser predictores de las puntuaciones en depresión, introduciendo ésta como variable dependiente.

A partir del análisis de correlación de Pearson, para averiguar si el nivel de depresión influye en alguno o algunos factores de autoconcepto se distribuyó la muestra en dos grupos: un grupo estaba integrado por los participantes con puntuaciones en depresión ausente, inferior a $5(n=25)(20$ mujeres, $80 \%$, y 5 hombres, $20 \%$ ). El otro grupo incluyó a quienes puntuaban por encima de este valor $(n=26)$ (16 mujeres, $61.5 \%$, y 10 hombres, 38.4\%). Tras asegurar el cumplimiento de los supuestos de la técnica de Kolmogorov-Smirnoff, $Z=.860, p=.450$, se realizó un MANCOVA para comparar las puntuaciones obtenidas por los dos grupos en depresión con las obtenidas en los distintos factores de autoconcepto. Puesto que se había constatado la relación de la edad con la variable depresión, se introdujo aquella como covariante. Además, para estimar el tamaño del efecto se calculó el índice $\eta^{2}$.

\section{Resultados}

El análisis de correlación de Pearson (Tabla 2) mostró correlaciones significativas, positivas y moderadas entre depresión y edad, sugiriendo que a más edad, puntuaciones más altas en depresión. También se hallaron correlaciones significativas, aunque negativas y moderadas, con autoconcepto físico y personal, esto es, mayores niveles de depresión se asociaban a menores puntuaciones en los dos factores señalados. Asimismo, se hallaron correlaciones significativas positivas entre estimación del $\mathrm{n}^{\circ}$ de personas de apoyo y autoconcepto físico y personal, sugiriendo que las personas que se sentían más apoyadas obtenían puntuaciones más altas en los dos factores, si bien las correlaciones no son de magnitud elevada. En cuanto a los años transcurridos desde la detección de la pérdida visual no se halló relación significativa con ninguna variable. En la Tabla 3 se muestran las medias y desviaciones estándar relativas a las variables mencionadas.

Tabla 2. Correlaciones de Pearson entre los diferentes factores del autoconcepto, edad, estimación del número de personas de las que dispone para que le presten ayuda, tiempo desde la detección de la pérdida visual y depresión.

$\begin{array}{lll}\text { Variables } & \text { NPA } & \text { ADPV Depresión }\end{array}$




\begin{tabular}{lllc}
\hline A. Físico & $.33^{*}(p=.04)$ & $.33(p=.05)$ & $-.48^{* *}(p=.00)$ \\
A. Moral-Ético & $.29(p=.07)$ & $.22(p=.19)$ & $-.23(p=.16)$ \\
A. Personal & $.36 *(p=.02)$ & $.29(p=.08)$ & $-.32 *(p=.04)$ \\
A. Familiar & $.30(p=.06)$ & $.24(p=.15)$ & $-.31(p=.05)$ \\
A. Social & $.19(p=.23)$ & $.16(p=.34)$ & $-.27(p=.08)$ \\
Edad & $-.01(p=.94)$ & $.27(p=.09)$ & $.40 *(p=.01)$ \\
\hline
\end{tabular}

Nota 1. A. Físico = Autoconcepto Físico; A. Moral-Ético $=$ Autoconcepto Moral-Ético; A. Personal = Autoconcepto Personal; A. Familiar $=$ Autoconcepto Familiar; A. Social $=$ Autoconcepto Social; NPA $=\mathrm{N}^{\circ}$ de Personas de Apoyo; ADPV = Años desde la Detección de la Pérdida Visual

Nota 2. * La correlación es significativa al nivel .05 (bilateral).

Nota 3. ** La correlación es significativa al nivel .01 (bilateral).

Tabla 3. Puntuaciones medias y desviaciones estándar de las variables de edad, depresión, factores de autoconcepto y $\mathrm{n}^{\circ}$ de personas de apoyo en el grupo completo y en los grupos por sexo.

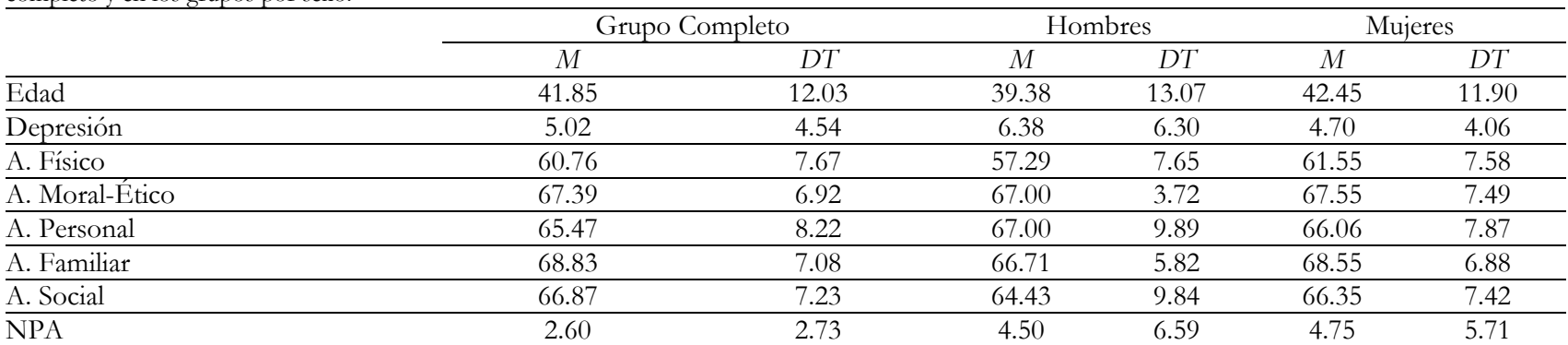

Nota. A. Físico $=$ Autoconcepto Físico; A. Moral-Ético $=$ Autoconcepto Moral-Ético; A. Personal = Autoconcepto Personal; A. Familiar $=$ Autoconcepto Familiar; A. Social $=$ Autoconcepto Social; NPA $=\mathrm{N}^{\circ}$ de Personas de Apoyo

El análisis de regresión lineal efectuado reveló diferencias estadísticamente significativas en el factor autoconcepto físico, sugiriendo que éste podría predecir el nivel de depre- sión, en una ecuación que explica el $54.207 \%$ de la varianza (Ver Tabla 4).

Tabla 4. Regresión lineal para la predicción de la depresión y los diferentes factores del autoconcepto.

\begin{tabular}{|c|c|c|c|c|c|c|c|c|}
\hline \multirow{2}{*}{ Variable dependiente } & \multirow{2}{*}{ Variables predictoras } & \multirow{2}{*}{ Beta } & \multirow{2}{*}{$t$} & \multirow{2}{*}{ Sig. } & \multirow{2}{*}{ Error } & \multicolumn{2}{|c|}{$I C$} & \multirow{2}{*}{$\%$ varianza } \\
\hline & & & & & & Mín. & Máx. & \\
\hline \multirow{5}{*}{ Depresión } & A. Físico & -.51 & -2.79 & .00 & .10 & -.50 & -.07 & 54.20 \\
\hline & A. Moral-Ético & -.07 & -.45 & .65 & .10 & -.25 & .16 & 19.72 \\
\hline & A. Personal & -.02 & .10 & .91 & .12 & -.23 & .25 & 12.26 \\
\hline & A. Familiar & -.06 & -.46 & .64 & .09 & -.24 & .15 & 8.98 \\
\hline & A. Social & -.04 & -.25 & .80 & .09 & -.21 & .17 & 4.81 \\
\hline
\end{tabular}

Nota. A. Físico = Autoconcepto Físico; A. Moral-Ético $=$ Autoconcepto Moral-Ético; A. Personal = Autoconcepto Personal; A. Familiar $=$ Autoconcepto Familiar; A. Social $=$ Autoconcepto Social.

Finalmente, los resultados del MANCOVA llevado a cabo indicaron la existencia de diferencias significativas entre los grupos, revelando que las personas con niveles más altos en depresión obtenían puntuaciones más bajas en todos los factores de autoconcepto, con magnitudes de $\eta^{2}$ que oscilan entre $50 \%$ y $87 \%$ de la varianza, siendo en el factor autoconcepto moral-ético en el que la magnitud $\eta^{2}$ resultó más relevante (Ver Tabla 6). En la Tabla 5 se presentan las medias y desviaciones estándar de las variables aludidas. 
Tabla 5. Puntuaciones medias y desviaciones estándar de las variables de edad, depresión, factores de autoconcepto y $\mathrm{n}^{\circ}$ de personas de apoyo, en hombres y mujeres, en ambos grupos.

\begin{tabular}{|c|c|c|c|c|c|c|c|c|}
\hline & \multicolumn{4}{|c|}{ Grupo $<5.00$} & \multicolumn{4}{|c|}{ Grupo $>5.00$} \\
\hline & \multicolumn{2}{|c|}{ Hombres } & \multicolumn{2}{|c|}{ Mujeres } & \multicolumn{2}{|c|}{ Hombres } & \multicolumn{2}{|c|}{ Mujeres } \\
\hline & $M$ & $D T$ & $M$ & $D T$ & $M$ & $D T$ & $M$ & $D T$ \\
\hline Edad & 31.33 & 5.68 & 39.71 & 12.73 & 44.20 & 14.32 & 43.83 & 11.72 \\
\hline Depresión & .33 & .57 & 1.59 & 1.58 & 10.00 & 5.05 & 9.00 & 3.04 \\
\hline A. Físico & 59.00 & .00 & 64.12 & 7.94 & 56.60 & 9.26 & 57.20 & 6.23 \\
\hline A. Moral-Ético & 67.50 & 4.95 & 69.47 & 5.68 & 66.40 & 3.78 & 63.60 & 9.02 \\
\hline A. Personal & 60.00 & 9.89 & 69.12 & 7.89 & 64.00 & 10.79 & 61.80 & 5.73 \\
\hline A. Familiar & 65.00 & 4.24 & 70.00 & 7.84 & 67.40 & 6.65 & 67.20 & 6.14 \\
\hline A. Social & 62.00 & 1.41 & 68.18 & 7.19 & 65.40 & 11.86 & 64.30 & 7.40 \\
\hline NPA & 3.00 & 3.46 & 5.82 & 7.02 & 5.40 & 8.20 & 3.64 & 4.22 \\
\hline
\end{tabular}

Nota. A. Físico = Autoconcepto Físico; A. Moral-Ético = Autoconcepto Moral-Ético; A. Personal = Autoconcepto Personal; A. Familiar $=$ Autoconcepto Familiar; A. Social $=$ Autoconcepto Social; $\mathrm{NPA}=\mathrm{N}^{\mathrm{o}}$ de Personas de Apoyo.

Tabla 6. MANCOVA de las puntuaciones obtenidas en depresión con los distintos factores de autoconcepto en los dos grupos.

\begin{tabular}{lll}
\hline & \multicolumn{1}{c}{$F$} & $\eta^{2}$ \\
\hline Intersección & $F(1,43)=60.86 ; p=.001$ & .93 \\
Autoconcepto Físico & $F(1,43)=101.14 ; p=.001$ & .50 \\
Autoconcepto Moral-Ético & $F(1,43)=181.53 ; p=.001$ & .87 \\
Autoconcepto Personal & $F(1,43)=121.77 ; p=.001$ & .83 \\
Autoconcepto Familiar & $F(1,43)=148.71 ; p=.001$ & .85 \\
Autoconcepto Social & $F(1,43)=114.18 ; p=.001$ & .82 \\
\hline
\end{tabular}

\section{Discusión}

El primer objetivo era conocer la existencia de relación entre alguno o algunos factores del autoconcepto, la estimación y percepción del número de personas con las que creen contar en caso de necesidad (por su vinculación con el autoconcepto y con la depresión), el nivel de depresión y la edad de adultos afectados por RP. Los resultados obtenidos revelan que a más edad los afectados obtienen puntuaciones más altas en depresión, dato coherente con las características de la enfermedad y acorde con el hallado por López-Justicia y Chacón-López (2015). Asimismo, se observa que mayor nivel de depresión se asocia a menores puntuaciones en los factores de autoconcepto físico y personal, siendo el primero de ellos el que mejor predice las puntuaciones en depresión (explicando el $54.2 \%$ de la varianza), como se deriva del análisis de regresión efectuado. Son datos que dejan entrever la percepción más negativa de esta población en ambos ámbitos, probablemente debido al efecto que ejerce en ellos el carácter progresivo y degenerativo de la patología visual.

Cuando se indaga en la relación con la estimación del número de personas de apoyo con las que los participantes creen contar se pone de manifiesto que quienes perciben mayor apoyo obtienen puntuaciones más altas precisamente en los factores de autoconcepto_físico y personal, llevando a suponer que el apoyo percibido parece contrarrestar el avance de la enfermedad haciendo que se sientan mejor justamente en factores relativos al aspecto físico y valoración de su personalidad. Este resultado es consonante con el hallado por Torres et al. (2010) en personas sin dificultades visuales, y con la investigación realizada por Reinhardt (2001), donde se subrayaba que la relación familiar y la ayuda proporcionada por los familiares se asociaban a una mejor adaptación a la pérdida de visión, lo que podría explicar el dato del presente estudio. No obstante, aun asumiendo el papel destacado que desempeñan los familiares, se ha alertado de que también requieren ayuda, dado el efecto emocional negativo que les causa la enfermedad de sus allegados con RP (Chacón-López, López-Justicia, et al., 2013; López-Justicia, Polo, et al., 2011).

El segundo objetivo del estudio es determinar si el nivel de depresión influye en alguno o algunos factores de su autoconcepto. Los resultados apuntan claramente en esta dirección, al poner de relieve que las personas con puntuaciones que entran en la categoría de depresión (por encima de 5) (Joffre-Velázquez, Martínez-Perales, et. al., 2007) se percibían más negativamente en todos los factores de autoconcepto (ver Tabla 5). Resultados que reflejan el malestar de los participantes en relación con el estado de salud, aspecto físico, valoración de la personalidad, situación familiar y estado moral/ético; probablemente debido a la ausencia de control sobre la condición visual progresiva, o la paulatina pérdida de autonomía funcional o sentido de auto-eficacia en un buen número de actividades de la vida diaria (Fuhr et al., 2007; Kiser y Dagnelie, 2008). El fracaso o estrés percibido en la práctica de estas habilidades podría tener una repercusión desfavorable en su estado emocional, de acuerdo con la teoría de depresión de Beck, Rush, Shaw y Emery (1987).

Si bien es cierto que el tipo de análisis llevado a cabo no permite probar que la causa de las puntuaciones más bajas en autoconcepto sea consecuencia de los niveles de depresión, sí se puede concluir que existe una relación clara entre ambas variables. Una mirada a la Tabla 5 permite observar que las personas con puntuaciones más altas en depresión obtienen peores resultados en cada uno de los factores de autoconcepto, hallándose éstos por debajo de la media en casi todos ellos, salvo en el familiar (67.40) y social (65.40) en los que los hombres se sitúan ligeramente por encima de la media, tal vez debido al efecto protector que ejerce la estimación de percepción de apoyo, algo mayor en el caso de los hombres (5.40) que en las mujeres (3.64).

A partir de estos datos creemos oportuno hacer hincapié en la importancia de la prevención de la depresión, para tra- 
tar de minimizar su repercusión negativa en el autoconcepto, en la funcionalidad visual, en los programas de rehabilitación visual en los que participen y en la calidad de vida de afectados por RP. No se olvide que de no prevenirla o tratarla existe el riesgo de incrementar sus niveles a medida que las personas afectadas avanzan en edad (López-Justicia y Chacón-López, 2015), pudiendo afectar negativamente a su autoconcepto. Asimismo se considera pertinente prestar más atención al desarrollo del autoconcepto desde los primeros años tras el diagnóstico de la enfermedad, máxime cuando otros estudios pusieron de relieve que el factor de autoconcepto familiar aparece alterado desde la adolescencia (LópezJusticia y Nieto 2006), situación que se mantiene en personas mayores (López-Justicia, Fernández-Castillo, et al., 2011). La propuesta de reforzar el autoconcepto es posible de llevar a la práctica al haberse resaltado que aunque el autoconcepto general parece mantenerse prácticamente inalterable, sin embargo, los específicos pueden variar a lo largo del tiempo como resultado de la experiencia en el medio social (Coll, 2002). Es obvio que la RP tiene un carácter progresivo y crónico, por lo que actualmente es imposible impedir su avance, pero es posible adoptar medidas de ayuda (en forma de adaptaciones de materiales, recursos tecnológicos y apoyo personal) que hagan viable el mantenimiento de la funcionalidad, percepción de competencia y autoeficacia de las personas afectadas; con el fin de que continúen participando activamente en todos los ámbitos de la vida laboral, personal, social, formativa, etc. No se puede ignorar que se ha vinculado el sentido de competencia personal a puntuaciones más altas en autoconcepto (Goñi et al., 2015; Madariaga y Goñi, 2009; Martinez y Sewel, 1996).

Se quiere señalar que el estudio tiene varias limitaciones.

\section{Referencias}

Agurtzane, M., y Vecino, E. (2009). Animal model and different therapies for treatment of Retinitis Pigmentosa. Histology and Histophatology, 24,1295-1322. http://www.hh.um.es/

Beck, A. T., Rush, A. J., Shaw, B. F., y Emery, G. (1979). Cognitive Therapy of Depression. New York: Guilford Press.

Beck, A. T., Rush, A. J., Shaw, B. F., y Emery, G. (1987). Cognitive Therapy of Depression (1 ${ }^{\text {st }}$ Ed.). NY: The Guilford Press.

Beck, A. T., Steer, R. A., y Carbin, M. G. (1988). Psychometric properties of the Beck Depression Inventory: Twenty-five years of evaluation. Clinical $\begin{array}{lll}\text { Psychology Review, } & \text { 8(1), 77-100. }\end{array}$ http://www.journals.elsevier.com/clinical-psychology-review/

Cattel, R. B., Eber, H. W., y Tatsuoka, M. M. (1970). Handbook for the sixteenn Personality factor Questioonaire. Illinois: Institute for Personality and Ability Testing.

Chacón-López, H., López-Justicia, M. D., Fernández, C., Chacón, A., y Polo, M. T. (2013). Emotional State of Family Members of Adults with Retinal Degeneration. Anales de Psicologia, 29(3), 772-778. DOI:10.6018/analesps.29.3.145181

Chacón-López, H., Pelayo, F. J., López-Justicia, M. D., Morillas, C. A., Ureña, R., Chacón-Medina, A., y Pino, B. (2013). Visual training and emotional state of people with Retinitis Pigmentosa. Journal of Rehabilitation $\begin{array}{lll}\text { Research and Development, } & \text { 50(8), } & 1157-1168 .\end{array}$ DOI:10.1682/JRRD.2012.06.0113

Coll, C. (2002). Constructivismo y Educación. En C. Coll, J. Palacios, y A. Marchesi, Desarrollo psicológico y educación, Vol. 2, Psicología de la educación escolar. Madrid: Alianza Editorial.
En primer lugar, para evaluar los niveles de depresión se ha recurrido a un cuestionario de auto-informe, en lugar de utilizar criterios convencionales, aunque conviene puntualizar que, aun no siendo una herramienta de diagnóstico para la depresión, ha sido confirmado como un buen método de cribado. Otro inconveniente radica en que se ha usado la versión abreviada, por lo que al disminuir el número de ítems también lo hace la fiabilidad; no obstante, es preciso señalar que se ha comprobado una correlación alta (.96) entre ambas versiones (Beck et al., 1979). Segundo, se ha evaluado una sola vez, impidiendo averiguar si los participantes tienen tendencia a mostrar un estado de ánimo depresivo, en cuyo caso serían más probables los síntomas depresivos como respuesta a la patología visual (Garaigordobil y Bernarás, 2009; McCrae y Costa, 1990). También se quiere señalar que el tamaño de la muestra es reducido y el número total de hombres y mujeres no es similar, lo que supone una limitación en la generalización de los resultados. Por otra parte es preciso tener en cuenta el carácter intencional del muestreo, debido a que todos los participantes eran voluntarios pertenecientes a dos asociaciones de afectados.

Finalmente, advertir de que, aun siendo conscientes del papel importante que juega el apoyo social percibido en el autoconcepto, no se ha evaluado con una escala convencional, sino que solo se introdujeron 2 ítems en la ficha de variables sociodemográficas, lo que podría explicar la ausencia de relación con el nivel de depresión, al contrario de lo señalado por Nunes et al. (2014). Situación que justifica la conveniencia de ahondar en estos resultados en futuros estudios, sobre todo por la importancia que parece desempeñar en la población referida.

Cubbigge, R.P. (2006). Campos visuales. Barcelona: Masson.

Fernández, E. (2007). Retinosis Pigmentaria: Preguntas y respuestas. Elche: Cátedra Bidons Egara.

Fernández, C., López-Justicia, M. D., y Polo, M. T. (2007). Discapacidad visible y no visible: diferencias en el autoconcepto. Estudios de Psicología, 28(3), 359-368. DOI: 10.1174/021093907782506498

Fierro, A. (2000). El desarrollo de la personalidad en la adultez y la vejez. En J. Palacios, A. Marchesi y C. Coll (Eds.), Desarrollo psicológico y educación, Vol. 1 (pp. 567-589). Madrid: Alianza.

Fitts, W. H. (1965). Tennessee Self-Concept Scale: Manual. Nashville, TN: Counselor Recording and Press.

Fuentes, M. C., García, J. F., Gracia, E., y Lila, M. (2011). Autoconcepto y ajuste psicosocial en la adolescencia. Psicothema, 23(1), 7-12. http://www.psicothema.com/

Fuhr, P. S. W., Liu, L., y Kuyk, T.K. (2007). Relationships between feature search and mobility performance in persons with severe visual impairment. Optometry and Vision Science, 84(5), 393-400. DOI:10.1097/OPX.0b013e31804f5afb

Garaigordobil, M., y Bernarás, E. (2009). Self-concept, self-esteem, personality traits and psychopathological symptoms in adolescents with and without visual impairment. The Spanish Journal of Psychology, 12(1), 149160. DOI: $10.1017 /$ S1138741600001566

Garanto, J. (1984). Las actitudes hacia sí mismo y su medición. En Temas de Psicología, $\mathrm{n}^{\circ}$ 7. Barcelona: Publicacions i Edicions de la Universitat de Barcelona. 
González-Pienda, J. A., Núñez, J. C., Álvarez, L., Roces, C., GonzálezPumariega, S., González, P., ... Bernardo, A. (2003). Adaptabilidad y cohesión familiar, implicación parental en conductas autorregulatorias, autoconcepto del estudiante y rendimiento académico. Psicothema, 15(3), 471-477. http://www.psicothema.com/

Goñi, E., Esnaola, I., Rodríguez, A., y Camino, I. (2015). Personal selfconcept and satisfaction with life in adolescence, youth and adulthood. Psicothema, 27(1), 52-58. http://www.psicothema.com/

Grant, P., Seiple, W., y Szlyk, J. P. (2011). Effect of depression on actual and perceived effects of reading rehabilitation for people with central vision loss. Journal of Rehabilitation Research and Development, 48(9), 1101-1108. DOI:10.1682/JRRD.2010.05.0080

Hahm, B., Shin, Y., Shim, E., Jeon, H., Seo, J., Cheng, H., y Yu, H. (2008). Depression and the vision-related quality of life in patients with Retinitis Pigmentosa. British Journal of Ophthalmology, 92(5), 650-654. DOI:10.1136/bjo.2007.127092

Hamel, C. (2006). Retinitis Pigmentosa. Orphanet Journal of Rare Diseases, 1, 40. DOI: $10.1186 / 1750-1172-1-40$

Harter, S. (1999). The construction of self: A developmental perspectives. NY: The Guilford Press.

Horowitz, A., y Reinhardt, J.P. (2006). Adequacy of the mental health system in meeting the needs of adults who are visually impaired. Journal of Visual Impairment and Blindness, 100, 871-874. http://www.afb.org/jvib/jvib_main.asp

Joffre-Velázquez, V. M., Martínez-Perales, G., García-Maldonado, G., y Sánchez-Gutiérrez, L. (2007). Depresión en estudiantes de medicina. Resultados de la aplicación del inventario de depresión de Beck en su versión de 13 ítems. Alcmeon Revista Argentina de Clinica Neuropsiquiatrica, 14(1), 86-93. http://www.alcmeon.com.ar/index.html

Kiser A., y Dagnelie, G. (2008). Reported effects of non-traditional treatments and complementary and alternative medicine by Retinitis Pigmentosa patients. Clinical and Experimental Optometry, 91(2), 166-176. DOI: $10.1111 /$ j.1444-0938.2007.00224.x

López-Justicia, M. D., y Chacón-López, H. (2015). Cambios emocionales asociados a la edad y género en personas con degeneración retiniana. Revista Argentina de Clínica Psicológica, 24(1), 57-66. http://www.clinicapsicologica.org.ar/

López-Justicia, M. D., Fernández-Castillo, A., Fernández, C., y Polo, T. (2011). Age, educational level and gender in self-concept of people with Retinitis Pigmentosa, Anales de Psicología, 27(2), 292-297. http://www.um.es/analesps/

López-Justicia, M. D., y Nieto, I. (2006). Self-concept of Spanish young adults with RP. Journal of Visual Impairment and Blindness, 100, 366-370. http://www.afb.org/jvib/jvib_main.asp

López-Justicia, M. D., Polo, T., Fernández, C., Chacón-López, H., DíazBatanero, C., y Chacón-Medina, A. (2011). Depresión y ansiedad en personas con retinosis pigmentaria, sus familiares y un grupo externo. $\begin{array}{lll}\text { Universitas } & \text { Psychologica, } & \text { 10(2), 467-476. }\end{array}$ http://revistas.javeriana.edu.co/sitio/psychologica/sccs/int.php?id=1 SICI: 1657-9267(201108)10:2<467:DAEPRP>2.0.TX;2-K

Madariaga, J. M., y Goñi, A. (2009). El desarrollo psicosocial. Revista de Psicodidáctica, 14(1), 95-118. DOI: 10.1387/RevPsicodidact.253
Marhs, H. W., Parada, R. H., y Ayotte, V. (2004). A multidimensional perspective of relations between self-concept (Self-Description Questionnaire II) and adolescent mental health (Youth Self- Report). Psychological Assessment, 16(1) 27-41. http://www.apa.org/pubs/journals/pas/

Martínez, R., y Sewell, K. (1996). Self-concept of adults with visual impairments. Journal of Rehabilitation, 62(2), 55-59. https://www.questia.com/library/p5155/the-journal-of-rehabilitation

Martos, M. J., y Muñoz, C. (2011). Apoyo funcional vs. disfuncional en una muestra de pacientes crónicos. Su incidencia sobre la salud y el cumplimiento terapéutico. Anales de Psicología, 27(1), 47-57. http://www.um.es/analesps/

McCrae, R. R., y Costa, P. T. (1990). Personality in Adulthood. NY: The Guilford Press.

Nunez, M., Munhoz, A., y Ferrari, H. (2014). Depression, family support and hopelessness: a correlated study. Universitas Psychologica, 13(2), 693702. DOI: 10.11144/Javeriana.UPSY13-2.dfsh

Organización Mundial de la Salud. (2006). Conquering depression. Mental health and substance abuse. http://www.who.int

Reinhardt, J. P. (2001). Effects of positive and negative support received and provided on adaptation to chronic visual impairment. Applied Developmental Science, 5(2), 76-85. DOI: 10.1207/S1532480XADS0502_3

Rundquist, J. (2004). Low vision rehabilitation of Retinitis Pigmentosa. Journal of Visual Impairment and Blindness, 98,718-724. http://www.afb.org/jvib/jvib_main.asp

Sanz, J., y Vázquez, C. (1998). El Inventario para la Depresión de Beck (BDI) como instrumento para identificar sujetos deprimidos y no deprimidos en la investigación psicopatológica: Fiabilidad, validez y datos normativos en muestras universitarias. Psicothema, 10(2), 303-318. http://www.psicothema.com/

Shavelson, J., Hubner, J. J., y Stanton, G. C. (1976). Self-concept: validation and construct interpretations. Review of Educational Research, 46(3), 407442. DOI:10.3102/00346543046003407

Smith, E., Nolen-Hoeksema, S., Fredrickson, B., y Loftus, G. (2003). Introducción a la Psicologia. Madrid: Thomson.

Szlyk, J. P., Seiple, W., Fishman, G. A., Alexander, K. R., Grover, S., y Mahler, C. L. (2001). Perceived and actual performance of daily tasks: relationship to visual function tests in patients with Retinitis Pigmentosa. Ophthalmology, 108, 65-75. DOI: 10.1016/S0161/6420(00)00413-9

Torres, G. F., Pompa, E. G., Meza, C. P., Ancer, L. E., y González, M. T. (2010). Relación entre autoconcepto y apoyo social en estudiantes universitarios. International Journal of Good Conscience, 5(2), 298-307. http://www.daenajournal.org/

Vázquez, C., y Sanz, J. (1997). Fiabilidad y valores normativos de la versión española del Inventario para la Depresión de Beck de 1978. Clínica y Salud, 8(3), 403-422. http://www.copmadrid.org/webcopm/resource.do?recurso $=4000$

Veltet, S., Gasper, R., Eisenacher, E., y Wittinghofer, A. (2008). The Retinitis Pigmentosa 2 gene product in a GTPase-activating protein for Arflike 3. Nature Structural \& Molecular Biology, 15(4), 373-380. DOI:10.1038/nsmb.1396

Zung, W. W. K. (1965). A Self-rating depression scale. Archives of General Psychiatry, 12, 63-70.

(Articulo recibido: 22-01-2015; revisado: 04-02-2015; aceptado: 16-09-2015) 